Size, composition and daily activity patterns

of white-lipped peccary groups (Tayassu pecari)

in Mirador-Río Azul National Park, Guatemala

\title{
Tamaño, composición y patrones diarios de actividad de grupos de pecarí de labios blancos (Tayassu pecari) en el Parque Nacional Mirador-Río Azul, Guatemala
}

\author{
José Fernando Moreira-Ramírez ${ }^{1 *}$, Jorge Erwin López ${ }^{2}$, Rony García-Anleu ${ }^{3}$, Francisco Córdova ${ }^{4}$ Tomás Dubón ${ }^{3}$

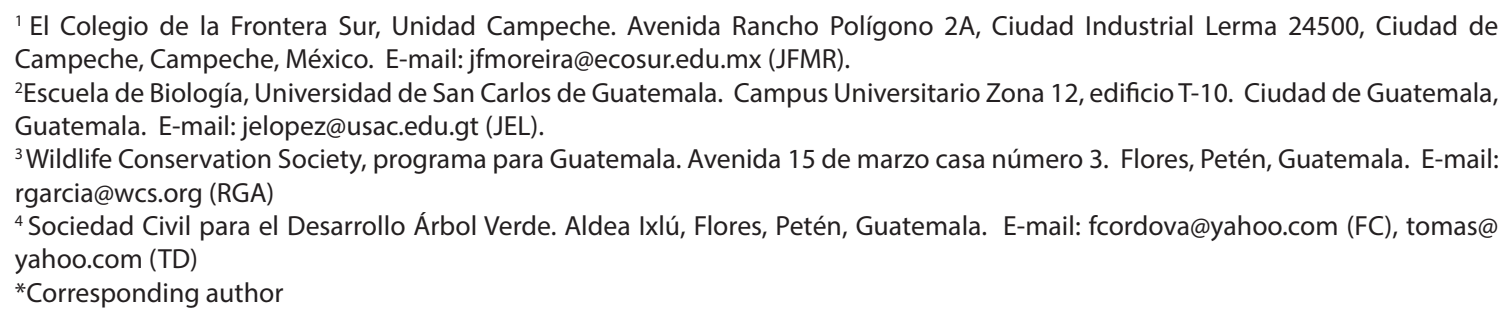

Introduction: The white-lipped peccary is a highly social species that forms large, cohesive groups of 10 to over 300 individuals. They are vulnerable to human presence and habitat fragmentation because they require large extensions of undisturbed forest with sufficient availability of food to maintain their biological requirements. This species has disappeared in $84 \%$ of its historic range in Guatemala. In Guatemala, the only place containing a viable population of white-lipped peccary is the Maya Biosphere Reserve although it is possible that residual populations exist in Punta de Manabique Wildlife Refuge, Laguna Lachuá National Park and Maya Mountains Biosphere Reserve. The specific objectives of this study were to describe the size and composition of white-lipped peccary groups in Mirador-Rio Azul National Park, a protected area within the Maya Biosphere Reserve, and describe their daily activity patterns at waterholes using camera traps and direct observations.

Methods: For analysis with camera traps we used photo-captures of white-lipped peccary obtained in a Rapid Ecological Assessment conducted in Mirador-Río Azul National Park in 2004. In addition, as part of this study, in 2006 we studied 12 waterholes, from April 22 to May 25. In 2007 direct observations were also made on waterholes from 8:00 at 17:00 during the months of February to May 2007 (dry season), and opportunistic encounters occurred on roads during June to January (rainy season). When a group was observed we recorded the date, time, number of newborns, juveniles and adults.

Results: In 2007 we recorded 11 groups of white-lipped peccary, five in waterholes and six in roads totaling 344 individuals. The estimated average of groups during the dry season in the Mirador-Río Azul National Park, was larger compared with Calakmul Biosphere Reserve and Corcovado National Park although these differences were not statistically significant $(U=9, P=0.08, U=31.5, P=0.63)$. Group composition included $94.2 \%$ adults, $3.8 \%$ juveniles, and $2 \%$ newborns. The groups visited waterholes more frequently between 8:00 and 12:00 .

Discussion and conclutions: The average group size of white-lipped peccary reported during the dry season in Mirador-Río Azul National Park is larger than the estimates reported in studies at the Calakmul Biosphere Reserve in Mexico and the Corcovado National Park in Costa Rica. This may be due to the moderate level of conservation within the Mirador-Río Azul National Park, high connectivity to protected areas of Mexico and Belize, and high availability of water for the presence of the Río Azul, which provides surface water availability, even during the dry season. White-lipped peccaries visited waterholes mainly during the day, registering two peaks of visits between 8:00 and 12:00. It is necessary to increase the protection in the Mirador-Río Azul National Park, especially at the borders with 
Mexico and Belize, and coordinate actions should be implemented in Mexico, Belize and Guatemala to preserve the natural heritage of these protected areas.

Key words: Camera traps, direct observations, Maya Forest, ungulate, waterholes.

\section{Introducción}

Los pecaríes pertenecen a la familia Tayassuidae, distribuida solamente en América y representada por tres especies (Sowls 1984; Grubb 2005). En Guatemala, se distribuyen dos especies: el pecarí de labios blancos (Tayassu pecari) y el pecarí de collar (Pecari tajacu; Taibel 1977; Reid 1997). El pecarí de labios blancos es el único ungulado social del Neotrópico que se desplaza en grupos grandes y cohesivos, generalmente de 10 a más de 300 individuos, en el interior de bosques tropicales densos (Sowls 1997; Fragoso 1998; Altrichter et al. 2001; Keuroghlian et al. 2004; Reyna-Hurtado et al. 2009). Ecológicamente, funge como importante dispersor y depredador de semillas. Sin embargo, es altamente susceptible a enfermedades infecciosas transmitidas por animales domésticos, a la presencia de seres humanos, quienes pueden cazarlo por ser una fuente importante de proteína para las comunidades rurales, y a la fragmentación de su hábitat (Sowls 1984; Morales 1993; Roling 1995; Bodmer et al. 1997; Fragoso 1997; Baur 1999; Altrichter y Boaglio 2004; Beck 2004; Reyna-Hurtado y Tanner 2005, 2007; Reyna-Hurtado et al. 2009). Por los motivos anteriores, esta especie se encuentra en la Lista de Especies Amenazadas de Guatemala en la Categoría tres (Manejo especial, uso controlado, CONAP 2000), en el apéndice Il del Convenio sobre Comercio Internacional de Especies Amenazadas de Fauna y Flora Silvestre (CITES 2006), y como Vulnerable por la Lista Roja de la Unión Mundial para la Naturaleza en todo su ámbito de distribución (Keuroghlian et al. 2013). Actualmente, esta especie ha desaparecido en un 84 \% de su distribución histórica en Guatemala (Altrichter et al. 2012). Se considera que en este país, el último lugar donde aún permanece una población viable de pecarí de labios blancos ( 3,500 individuos) es dentro de la Reserva de la Biosfera Maya (RBM; Traill et al. 2007; WCS-CONAP 2009; Altrichter et al. 2012) y posiblemente existan poblaciones muy reducidas en el Refugio de Vida Silvestre Punta de Manabique, Parque Nacional Laguna Lachuá y Reserva de la Biosfera Montañas Mayas (WCS-CONAP 2009). También en varias áreas protegidas ubicadas al sur de la Reserva de la Biosfera Maya RBM, el pecarí de labios blancos presenta bajas abundancias por la alta presión de caza. De esta manera, se le considera extinto ecológicamente. Por ejemplo, en la Ecorregión Lachuá, cazadores mencionan que esta especie es cada vez más escasa, y en la Reserva de la Biosfera Sierra de las Minas, no se tienen registros de ella desde hace más de 20 años (Moreira-Ramírez 2004; Rosales-Meda et al. 2010).

Con más de dos millones de hectáreas, la RBM cubre más de la mitad del departamento de Petén y casi una quinta parte del territorio de Guatemala. Junto con áreas protegidas de México y Belice, forma la Selva Maya, el mayor bloque continuo de bosque tropical ubicado al norte del Amazonas (Sanderson et al. 2002; Radachowsky et al. 2012).

En Guatemala existe un vacío de información sobre aspectos ecológicos y biológicos básicos de la historia natural del pecarí de labios blancos. Este estudio es el único que se ha enfocado en conocer los tamaños, composición y patrones diarios de actividad de grupos de esta especie. Otros estudios se han enfocado en caracterizar la actividad de cacería en comunidades cercanas o en áreas protegidas. El pecarí de labios blancos es una de las especies más cazadas (Morales 1993; Roling 1995; Baur 1999; Rosales-Meda et al. 2010).

El presente estudio se desarrolló en el Parque Nacional Mirador-Río Azul, ubicado dentro de la RBM. Allí, las poblaciones de pecarí de labios blancos se encuentran menos afectadas por las actividades humanas que en cualquier otra parte del país (WCS-CONAP 2009). Los objetivos 
específicos de la presente investigación fueron describir el tamaño y la composición de los grupos de pecarí de labios blancos, así como los patrones diarios de su actividad en aguadas. Se recurrió a trampas-cámara y a observaciones directas. Como hipótesis, se planteó que, debido al alto grado de conservación del Parque Nacional Mirador Río Azul, los tamaños de los grupos de pecarí de labios blancos en la época seca serán mayores comparados con los del Parque Nacional Corcovado, Costa Rica. Debido a la presencia de bosques más húmedos, los grupos serán mayores comparados con los de la Reserva de la Biosfera Calakmul, en México. Los datos de esta investigación ayudarán al desarrollo de planes para conservar esta especie en la Reserva de la Biosfera Maya y en la Selva Maya.

\section{Materiales y métodos}

Área de estudio. El Parque Nacional Mirador-Río Azul (PNMRA) se localiza en la esquina noreste de la Reserva de la Biosfera Maya, entre las coordenadas $17^{\circ} 44^{\prime} 31^{\prime \prime} \mathrm{N},-89^{\circ} 34^{\prime} 32^{\prime \prime} \mathrm{W}$. Limita al oeste con la concesión industrial de Paxbán y al este con Belice. Al norte, se encuentra la Reserva de la Biosfera de Calakmul, en México, y al sur, la comunidad de Uaxactún (CONAP y ONCA 2002; Figura 1).

El área fue declarada Parque Nacional en 1990, con el establecimiento de la Reserva de la Biosfera Maya. El área total del PNMRA es de 116,911 hectáreas (CONAP 1995, CONAP y ONCA, 2002) y se encuentra cubierta por Bosque Húmedo Subtropical Cálido, clasificado con base en el Sistema de Holdridge (De la Cruz 1982). Anualmente, la temperatura varía de 22 a $34{ }^{\circ} \mathrm{C}$. La precipitación promedio anual es de $1,323 \mathrm{~mm}$. La altitud varía entre $250 \mathrm{msnm}$ al oeste y 100 msnm al este (CONAP y ONCA 2002; Moreira-Ramírez 2009). Existen dos épocas marcadas: la época seca abarca de febrero a mayo, y la época lluviosa, de junio a enero. En la Selva Maya, los suelos son de origen calcáreo. Esto ocasiona que el agua de lluvias se filtre rápidamente y no existan cuerpos de agua permanentes. Sin embargo, hay zonas donde las características del suelo permiten la acumulación de la precipitación, lo que forma cuerpos de agua conocidos localmente como aguadas (Reyna-Hurtado et al. 2010a). Las aguadas son sumamente importantes para la vida silvestre en este parque, ya que proveen de agua superficial a diferentes especies, entre ellas al pecarí de labios blancos (Moreira-Ramírez 2009; Moreira-Ramírez y García 2011).

Tamaño y composición de grupos. Para registrar el tamaño y la composición de los grupos, fueron visitadas 12 aguadas entre las 8:00 h a 17:00 h, por un periodo de 10 días durante cada mes. Se abarcó la época seca comprendida entre de febrero a mayo de 2007. Además, durante la época lluviosa, entre junio y octubre de 2007, se realizaron conteos oportunos de grupos de pecaríes de labios blancos en caminos (Tabla 1). El tamaño y la composición de los grupos fueron obtenidos por medio de registros realizados tanto en aguadas como en caminos. Se abarcó la época seca y lluviosa (Tabla 1). Las observaciones en aguadas fueron realizadas en árboles a una altura aproximada de $4 \mathrm{~m}$ para contar el tamaño mínimo de los grupos. Las observaciones en caminos fueron realizadas cuando nos desplazábamos del campamento hacia las aguadas. En el momento de observar un grupo, se anotó la fecha, hora, número de crías, juveniles y adultos. Para identificar crías, juveniles y adultos, se utilizaron los siguientes criterios: las crías son de tamaño pequeño comparado con juveniles y adultos; su color es café claro y se desplazan junto a sus madres. Los juveniles presentan tamaños intermedios y son de color café claro negruzco. Los adultos son de color negro y presentan el mentón blanco (Sowls 1984, 1997).

Patrones diarios de actividad en aguadas. Para describir los patrones diarios de actividad del pecarí de labios blancos en las aguadas, se utilizaron trampas-cámara y se recurrió a observaciones directas. Para el análisis con trampas-cámara, se utilizaron fotocapturas de pecarí de labios blancos obtenidas en la Evaluación Ecológica Rápida realizada en el PNMRA en 2004 
(García y Radachowsky 2004). Asimismo, como parte de este estudio, en 2006 se estudiaron 12 aguadas del 22 de abril al 25 de mayo. En cada aguada se colocó una trampa-cámara de film de 35 mm, modelo Camtrakker ${ }^{\circledR}$, con cámaras marca Olympus, Canon y Yashica (Camtrak South, 1050 Industrial Drive, Watkinville, GA 30677 USA). Solamente en la aguada El Infierno Dos, fueron colocadas dos trampas-cámara. El tiempo de muestreo durante 2006 fue de 33 días por trampa-cámara. El esfuerzo de muestreo fue de 396 trampas por noche. Durante 2007, se estudiaron seis aguadas en febrero. En cada aguada se colocó una trampa-cámara de film de $35 \mathrm{~mm}$ modelo Leaf River ${ }^{\mathrm{TM}}$ C-1BU, con cámara Canon@ Sure Shot Owl (Leaf River Outdoor Products, Taylorsville, MS 39168 USA). El tiempo de muestreo durante este año fue de 24 días por trampa-cámara. El esfuerzo de muestreo fue de 144 trampas por noche. Las trampas-cámaras se programaron para tomar fotografías durante 24 horas. Se activó la trampa-cámara debido a un cambio de temperatura o movimiento. Se tomó una fotografía por minuto. Cada trampa-cámara fue programada para registrar en cada fotografía el día y la hora. Las trampas-cámara fueron colocadas a una altura promedio de $56 \mathrm{~cm}(50-60 \mathrm{~cm})$ y fueron revisadas cada 10 a 15 días. Al final de este lapso, se cambiaron los rollos (35mm ASA 400 de 24 exposiciones marca Konica Minolta) y baterías (Energizer $\odot$ ). Las observaciones directas en las aguadas se realizaron de las 8:00 h a las 17:00 h de febrero a mayo de 2007. Durante estas observaciones se anotó la hora en que cada grupo visitó la aguada.

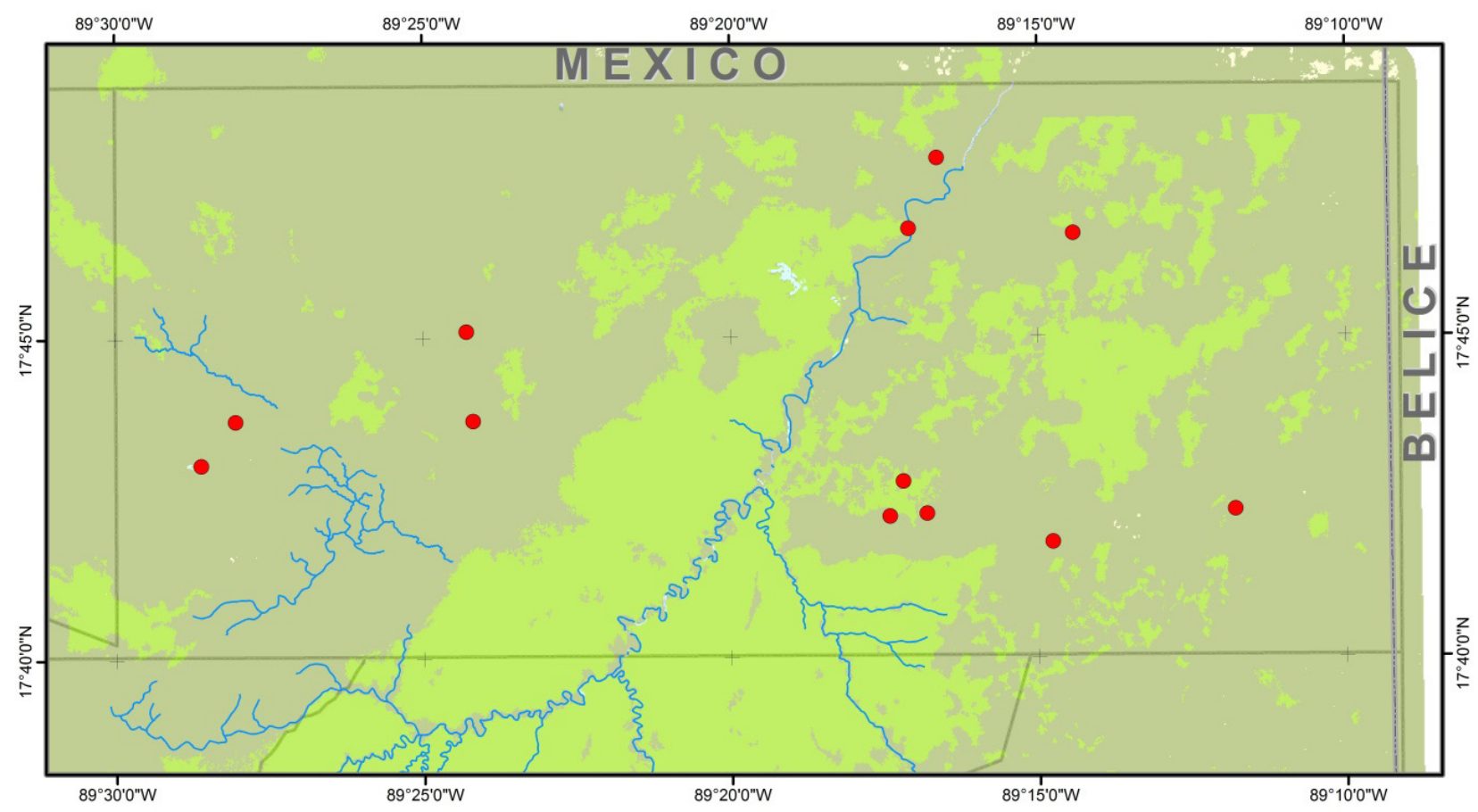

Ubicación Regional

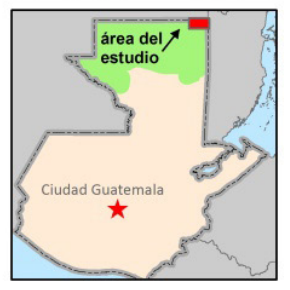

\section{Leyenda:}
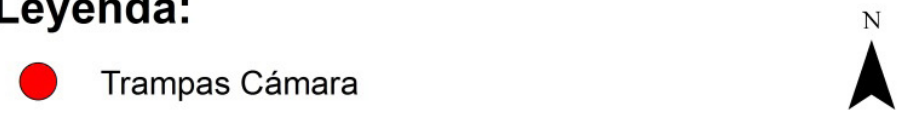

Bosque latifoliado bajo

Bosque latifoliado medio-alto

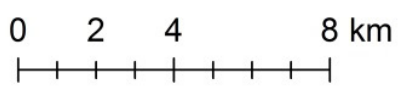

Figura 1. Mapa de ubicación geográfica del Parque Nacional Mirador - Río Azul y ubicación de las trampas cámaras en 12 aguadas, Guatemala. 


\section{Análisis de los datos}

Tamaño y composición de grupos. Las variables medidas fueron el número de individuos (adultos, juveniles y crías) por grupo y el número total tanto en la época seca como en la lluviosa. Los descriptores de la composición de los grupos de pecarí de labios blancos se expresaron como porcentaje de adultos, juveniles y crías. Los descriptores de organización social fueron el tamaño de los grupos, la media del tamaño y la composición ( \pm desviación estándar) de grupos. Para comparar el tamaño de los grupos entre estaciones (Seca: febrero-mayo; Lluviosa: junio-enero) con datos de la Reserva de la Biosfera de Calakmul (RBC), México (Seca: diciembre-mayo; Reyna-Hurtado 2007) y del Parque Nacional Corcovado (PNC), Costa Rica (Seca: diciembre-abril, Estrada 2005), se utilizó una prueba U de Mann-Whitney con el programa R 2.13.1 (R Development Core Team 2011). Para probar las hipótesis, se compararon los tamaños de los grupos del presente estudio con los datos de la la RBC y del PNC. Se emplearon únicamente los registros de la época seca para los tres sitios.

Patrones diarios de actividad en aguadas. A fin de considerar un registro como fotocaptura independiente, cada imagen de pecarí de labios blancos tuvo que ser tomada como mínimo cada 30 minutos (O'Brein et al. 2003; Bowkett et al. 2007). Posteriormente, se agruparon las fotografías y observaciones directas en el programa Excel de Microsoft $\odot$ y se estimó la frecuencia de visitas a lo largo de 24 horas divididas en lapsos de dos horas (Cuellar y Noss 2003).

\section{Resultados}

Tamaño de grupos. Durante 2007, de las 12 aguadas visitadas, se obtuvieron registros del tamaño de grupo de pecarí de labios blancos en cinco de ellas. En seis ocasiones se registró la presencia de grupos de esta especie en caminos (Tabla 1). En total, se contabilizaron 344 individuos. En la época seca, se estimó una media de 39.8 ( \pm 14.4) individuos. En la época lluviosa se estimó una media de 24.2 ( \pm 8.4) individuos. Para estimar la media del tamaño de los grupos en el PNMRA a lo largo del año, se tomó la información de la época seca y de la lluviosa. La media del tamaño de grupo fue de 31.3 individuos ( \pm 13.6). El tamaño de los grupos tendió a ser mayor en época seca en comparación con los tamaños en la época lluviosa, aunque dicha diferencia no fue estadísticamente significativa $(U=23.5, P=0.14)$. Tras comparar el tamaño de los grupos registrados solo durante la época seca en el PNMRA y en la RBC (área sin cacería, Reyna-Hurtado 2007) con el PNC (Estrada 2005), se concluyó que los grupos del PNMRA (Media $=39.8 \mathrm{DE}=14.4, n=5$ ) fueron más grandes que los de la RBC (Media $=25.6 \mathrm{DE}=5.5, n=9$, Reyna-Hurtado 2007) y los del PNC (Media $=36 \mathrm{DE}=16.2, n=15$, Estrada 2005). Dichas diferencias, sin embargo, no fueron estadísticamente significativas $(U=9, P=$ $0.08, \mathrm{U}=31.5, P=0.63$ ).

Los grupos de pecarí de labios blancos están compuestos principalmente por adultos. Se encontró que, de un total de 11 grupos observados, las crías componen el 2\% (7) de la población, los juveniles componen el $3.8 \%$ (13) y los adultos el $94.2 \%$ (324). De febrero a octubre, en total se observaron tres grupos con crías. Dos de estas observaciones se realizaron en mayo y una en agosto (Tabla 1). Las dos observaciones de crías en mayo correspondían a gemelos. Se estima que los nacimientos se dan en abril y en julio, aproximadamente, en estos casos. Además, si tomamos en cuenta que el período de gestación es, en promedio, de 158 días (entre 156 y 162 días; Sowls 1984), y los nacimientos se dan con mayor frecuencia en mayo, el pecarí de labios blancos se aparea en diciembre.

Patrones diarios de actividad en aguadas. Durante la Evaluación Ecológica Rápida realizada en el PNMRA en 2004, se obtuvieron 13 fotocapturas de pecarí de labios blancos (García y Radachowsky 2004). En 2006 y 2007, se obtuvieron 27 y nueve fotocapturas respectivamente. Asimismo, en 2007 se registraron cinco observaciones directas de grupos de esta especie en las aguadas. Los patrones diarios de actividad del pecarí de labios blancos en las aguadas fueron principalmente 
diurnos. El $57 \%$ (28) de las fotocapturas se registraron entre las 8:00 h y las 12:00 h. Los picos de actividad se dieron entre 8:00 y 12:00. También se registró una leve actividad en las aguadas durante la noche, entre las 18:00 y 00:00 (Figura 2).

Tabla 1. Localidad, sitio, época, fecha, tamaño y composición de grupos del pecarí de labios blancos, Tayassu pecari, en el Parque Nacional Mirador-Río Azul, Reserva de la Biosfera Maya, Guatemala.

\begin{tabular}{|c|c|c|c|c|c|c|c|}
\hline Localidad & Sitio & Época & Fecha & Crías & Juveniles & Adultos & Total \\
\hline El Cedro-Agua Salada & Aguada & Seca & $14 / 02 / 2007$ & 0 & 0 & 60 & 60 \\
\hline El Barranco & Aguada & Seca & $09 / 03 / 2007$ & 0 & 2 & 40 & 42 \\
\hline Tambitos & Aguada & Seca & $19 / 04 / 2007$ & 0 & 0 & 45 & 45 \\
\hline Tambitos & Aguada & Seca & $13 / 05 / 2007$ & 2 & 3 & 19 & 24 \\
\hline Mirador-Gardenias & Aguada & Seca & $13 / 05 / 2007$ & 2 & 5 & 21 & 28 \\
\hline Zacatalito & Camino & Lluviosa & $17 / 06 / 2007$ & 0 & 0 & 18 & 18 \\
\hline Brecha Sur 1 & Camino & Lluviosa & $15 / 07 / 2007$ & 0 & 3 & 29 & 32 \\
\hline Brecha Sur 2 & Camino & Lluviosa & $28 / 08 / 2007$ & 3 & 0 & 27 & 30 \\
\hline Dos Lagunas-El Cedro & Camino & Lluviosa & $16 / 09 / 2007$ & 0 & 0 & 28 & 28 \\
\hline El Cedro-Vergelito & Camino & Lluviosa & $14 / 10 / 2007$ & 0 & 0 & 27 & 27 \\
\hline Vergelito & Camino & Lluviosa & $30 / 10 / 2007$ & 0 & 0 & 10 & 10 \\
\hline Total & & & & 7 & 13 & 324 & 344 \\
\hline
\end{tabular}

\section{Discusión}

Esta investigación es la primera en Guatemala que estima el tamaño y la composición, así como los patrones diarios de actividad en aguadas y en época de nacimientos de los grupos de pecarí de labios blancos,

Tamaño de grupos. Encontramos que en el PNMRA, los grupos más grandes se registraron en la época seca. En esta época se registraron grupos de 60 y 45 individuos en febrero y abril, respectivamente. La media del tamaño de grupos en el PNMRA es una de las más grandes reportadas hasta ahora en Mesoamérica. Esta información debe tomarse con cautela debido al pequeño número de observaciones que realizamos. En la concesión forestal comunitaria de Carmelita, Zona de Usos Múltiples de la RBM, Baur (1999) se estimó una media de 50 individuos por grupo. La concesión forestal comunitaria de Carmelita se encuentra ubicada al oeste de la RBM. En esta parte de la reserva, el agua superficial es más abundante comparada con el PNMRA. El pecarí de labios blancos posee una alta afinidad con los lugares con presencia de agua, y permanece siempre en las periferias de ríos, salitrales y aguadas, sobre todo durante la época seca. Los sitios con mayor presencia de agua manifiestan mejor calidad de hábitat para esta especie en comparación con los lugares secos con baja precipitación (Reyna-Hurtado et al. 2009; Reyna-Hurtado et al. En revisión).

La media del tamaño de los grupos reportados durante la época seca en el PNMRA resulta más grande que las estimaciones reportadas en estudios en la RBC de México y en el PNC de Costa Rica (Reyna-Hurtado y Tanner 2005; Reyna-Hurtado et al., 2009, Estrada 2005; Reyna-Hurtado et al. 2010b). Lo anterior puede deberse al moderado nivel de conservación del PNMRA, a la 
alta conectividad con áreas protegidas de México y Belice, y a la alta disponibilidad de agua por la presencia del Río Azul, que brinda disponibilidad de agua superficial incluso durante la época seca. Se trata de un factor crítico para la especie (Reyna-Hurtado y Tanner 2005; Ramos et al. 2007; SalomPérez et al. 2007; Reyna-Hurtado et al. 2010). Los registros de grupos de pecarí de labios blancos en bosques secos de Argentina (Media = 23, intervalo de individuos entre 7 - 50; Altrichter 2005) son también menores a los reportados en el PNMRA. No obstante, al comparar las estimaciones de los grupos del PNMRA con registros obtenidos en Brasil, Bolivia y Perú, los tamaños en el PNMRA son menores. En estos tres países, los grupos de pecarí de labios blancos pueden estar integrados hasta por más de 100 individuos (Kiltie y Terborgh 1983; Fragoso 1998; Sowls 1997). En Brasil, existen reportes confirmados de grupos de más de 200 individuos (Fragoso 2004) y reportes anecdóticos de grupos que exceden los 1,000 individuos (Sowls 1997; Fragoso 1998). En la isla de Maraca, Brasil, y en el Parque Nacional Manu de Perú, se estimaron tamaños promedio de grupos de pecarí de labios blancos de 114 y 106 individuos, respectivamente (Fragoso 1998; Kiltie y Terborgh 1983). Los grupos tienden a ser menores en hábitats secos como la RBC, el Chaco (en el norte de Argentina) y los bosques secos de Venezuela, donde la precipitación promedio es baja. Otro factor importante que afecta negativamente el tamaño de los grupos es la actividad de cacería. Los grupos de esta especie tienden a ser más grandes en áreas protegidas en comparación con los sitios que presentan asentamientos humanos donde se practica la cacería de subsistencia. Es posible que esta relación sea un indicio de que las perturbaciones humanas tengan un efecto negativo sobre la especie (Kiltie y Terborgh 1983; March 1993; Reyna-Hurtado et al. en prensa). El pecarí de labios blancos es una especie muy vulnerable a la presencia humana y es poco frecuente observar grupos cercanos a las comunidades (Baur 1999; Reyna-Hurtado y Tanner 2005, 2007). Reyna-Hurtado y colaboradores (2009 y 2010) reportaron tamaños menores (en comparación con el centro de la RBC) de grupos de pecarí de labios blancos en sitios con cacería cercanos a los asentamientos humanos.

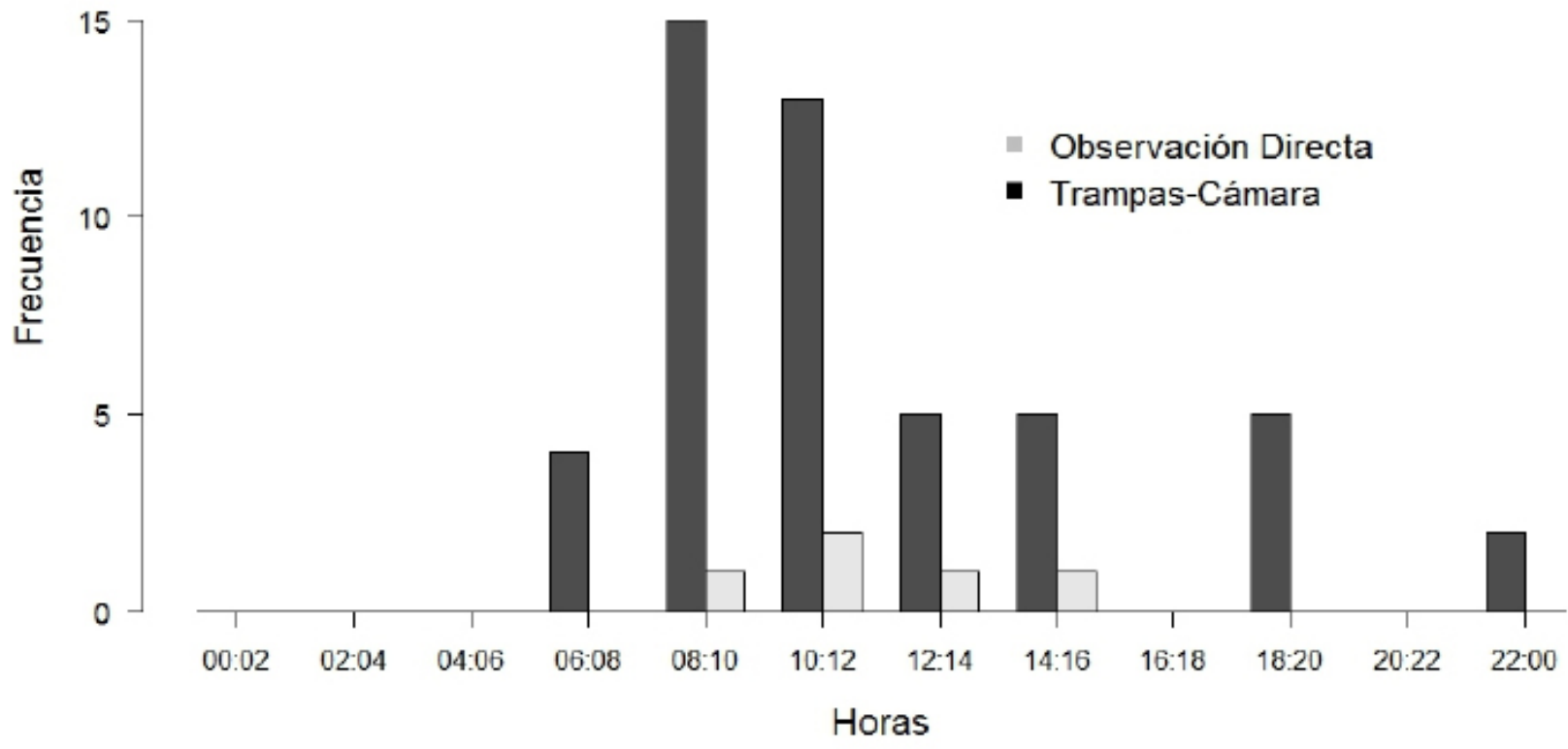

Figura 2. Patrones diarios de actividad de grupos de pecarí de labios blancos en la aguadas registrados por medio de trampascámara $(n=49)$ y observaciones directas $(n=5)$. 
EI PNMRA es una de las áreas protegidas mejor conservadas de Guatemala. Del lado de esta nación, el poblado más cercano se encuentra aproximadamente a $50 \mathrm{~km}$. La presión de cacería se realiza por incursiones de gente proveniente de este poblado. Cazan especies de alto valor cinegético y después venden su carne en la ciudad de Flores, el centro urbano turístico de la región. Reportes de guarda-recursos mencionan que en el parque hay incursiones ilegales de cazadores mexicanos que ingresan a acampar en el sitio llamado La Lagunita, al centro norte del PNMRA (J. M. Castillo, com. pers.). Para evitar estas incursiones en el parque, es necesario aumentar los patrullajes y el número de guarda-recursos, a fin de contar con mayor presencia en las áreas de la frontera norte con México y al este, con Belice (F. Asturias, com. pers.). Asimismo, es importante trabajar en conjunto con la administración de la RBC para aumentar la eficacia de los patrullajes fronterizos. Acciones como estas permitirán un mejor control en el parque y evitarán que especies tan vulnerables como el pecarí de labios blancos sigan siendo cazadas.

Composición de grupos y época de nacimientos. La proporción de adultos en grupos de pecarí de labios blancos fue similar en el PNMRA y en lo reportado en la Selva Lacandona y la RBC (Naranjo 2002; Reyna-Hurtado 2007). Más de $90 \%$ de los individuos que componen los grupos pertenecen a esta clase de edad. La proporción de juveniles y crías, en grupos del PNMRA fueron similares a lo reportado en la RBC. Representan menos de $10 \%$ de los individuos que componen los grupos.

La información obtenida en el presente estudio indica que la mayoría de los nacimientos se da a finales de la época seca, en mayo. Este mismo patrón de nacimientos durante la época seca ha sido reportado en la concesión forestal comunitaria de Carmelita. En contraste, los picos de nacimientos en la RBC se dan al inicio de la época seca de enero a marzo, principalmente (Reyna-Hurtado et al. 2009). En la concesión forestal comunitaria de Carmelita fueron registradas hembras preñadas en los meses de más lluvia (septiembre-octubre; Baur 1999). Si tomamos en cuenta que el período de gestación es en promedio de 158 días (entre 156 y 162 días; Sowls 1984), y que los nacimientos en el PNMRA se dan con mayor frecuencia en mayo, el pecarí de labios blancos se aparea durante noviembre y diciembre. Actualmente, la Ley General de Caza guatemalteca (Congreso de la República de Guatemala 2004) establece que esta especie puede ser cazada durante todo el año. Sin embargo, la información obtenida en el presente estudio en el PNMRA, en la RBC y en la concesión forestal comunitaria de Carmelita demuestra que esta especie presenta con mayor frecuencia nacimientos durante la época seca en la Selva Maya. Es importante tomar en cuenta la información anterior con el fin de modificar la época de cacería y el número de individuos por cosechar, o para evitar la caza de esta especie en áreas protegidas. Además, se le debe dar seguimiento a las licencias extendidas para que los cazadores cumplan con el número de individuos por cosechar, con la finalidad de evitar la extinción local de la especie. Instituciones de gobierno como el Consejo Nacional de Áreas Protegidas, investigadores, Organizaciones No Gubernamentales y comunitarias deben tomar esta iniciativa para desarrollar planes de manejo de esta especie.

Patrones diarios de actividad en aguadas a partir de datos de trampas-cámara y de observaciones directas. El pecarí de labios blancos es una especie principalmente diurna. Durante la época seca, sus movimientos se restringen a sitios con agua superficial (Reyna-Hurtado et al. 2009). Los grupos visitaron las aguadas principalmente durante el día. Se registraron dos picos de visitas entre las 8:00 y 12:00. Se ha reportado este mismo patrón en el bosque del Chaco y en la Chiquitanía, en Bolivia. Se utilizaron trampas-cámaras en salitrales (Maffei et al. 2002; Cuellar y Noss 2003). Los grupos de pecarí de labios blancos en el PNMRA visitaron las aguadas con menor frecuencia durante la noche, entre las 18:00 y 20:00. En el Parque Nacional Corcovado, los grupos fueron fotocapturados en caminos entre las 7:00 y 9:00, y durante la tarde, entre las 15:00 y 17:00. Por medio de observaciones directas en bañaderos, se reportaron visitas 
durante todo el día, entre las 8:00 y 16:00 (Estrada 2005). Los grupos visitaron las aguadas en un tiempo relativamente corto, entre 5 y 10 minutos. Solamente en mayo, cuando la sequía es más pronunciada y cuando la mayoría de las aguadas tienden a secarse, el tiempo de visita a las aguadas permanentes aumentó entre 40 a 50 minutos (Moreira-Ramírez 2009).

El uso de trampas-cámara es un método confiable, no invasivo, recomendable para estimar patrones diarios de actividad del pecarí de labios blancos y de otras especies de mamíferos y aves que usan sitios clave, como aguadas, durante determinada época.

Implicaciones para la conservación. En Guatemala, la RBM, junto con áreas protegidas de México y Belice, forma la Selva Maya, el bosque continuo tropical mejor conservado al norte de Colombia. Ha sido declarado como uno de los lugares más importantes para conservar la biodiversidad (Mittermeier et al. 1998, Myers et al. 2000; Sanderson et al. 2002). El PNMRA, con sus 116,911 hectáreas, es la piedra angular para la conectividad entre las áreas protegidas de México y Belice. Este parque se encuentra en una ubicación estratégica, que puede permitir el intercambio de individuos de varias especies amenazadas en la región.

Los grupos de pecarí de labios blancos en el PNMRA son relativamente grandes, en comparación con los de otras áreas protegidas de Mesoamérica. Esto demuestra la importancia de este parque para conservar la población de esta especie en Guatemala. Las poblaciones de pecarí de labios blancos han sido extirpadas localmente de otras áreas de este país, principalmente por la pérdida de hábitat y por la sobrecacería (WCS-CONAP 2009). Actualmente, la distribución de la especie ha desaparecido en un 84 \% de su distribución histórica en Guatemala. Posiblemente existen poblaciones muy reducidas en el Refugio de Vida Silvestre Punta de Manabique, Parque Nacional Lachuá, y en la Reserva de la Biosfera Montañas Mayas (WCS-CONAP 2009). En Guatemala, la RBM es el único lugar donde aún permanece una población viable de pecarí de labios blancos. Esta reserva contiene una población residente suficientemente grande para ser en potencia autosostenible durante los próximos 100 años, con un hábitat adecuado y una diversa base alimentaria (Altrichter et al. 2012). Las intervenciones de conservación deben incluir controles efectivos a fin de evitar la cacería y favorecer el ecoturismo en el PNMRA. Hay evidencias de que el pecarí de labios blancos utiliza grandes extensiones de territorio y se desplaza por varios hábitats (Fragoso 1998, ReynaHurtado et al. 2009). Debido a lo anterior, es necesario aumentar la protección en el PNMRA, sobre todo en las fronteras con México y Belice. También se deben coordinar acciones trinacionales para preservar el patrimonio natural de estas áreas protegidas.

\section{Agradecimientos}

A Wildlife Conservation Society, Programa para Guatemala, en especial a R. Balas McNab. A J. Soto, por permitirnos trabajar en este proyecto. Al Fondo Nacional de Ciencia y Tecnología, proyecto 11806, otorgado por la Secretaria de Ciencia y Tecnología, Consejo Nacional de Ciencia y Tecnología, Programa de Paisajes Vivientes de WCS-Guatemala, y a IdeaWild, por el soporte financiero y la donación de equipo. Al Consejo Nacional de Áreas Protegidas, Instituto de Antropología e Historia, y a la Asociación Balam, por el apoyo y las facilidades brindadas. A los técnicos de campo de WCSGuatemala, por toda su ayuda. A N. Estrada, por compartir los datos de su investigación. A R. ReynaHurtado, M. Briceño, por sus sugerencias y a N. Solís por la ayuda en la elaboración del mapa.

\section{Resumen}

Introducción: El pecarí de labios blancos es una especie altamente social, que forma grupos grandes y cohesivos de 10 y hasta más de 300 individuos. Es vulnerable a la presencia humana y a la fragmentación de su hábitat, ya que necesita grandes extensiones de bosque no perturbado con suficiente disponibilidad de alimento para mantener sus requerimientos biológicos. Actualmente, esta especie ha desaparecido en un $84 \%$ de su distribución histórica en 
Guatemala. En este país el único lugar donde aún permanece una población viable es dentro de la Reserva de la Biosfera Maya, posiblemente existan poblaciones muy reducidas en el Refugio de Vida Silvestre Punta de Manabique, Parque Nacional Laguna Lachuá y Reserva de la Biosfera Montañas Mayas. Los objetivos específicos de la presente investigación fueron describir el tamaño y composición de los grupos de pecarí de labios blancos en el Parque Nacional Mirador-Río Azul que se encuentra dentro de la Reserva de la Biosfera Maya, así como describir sus patrones diarios de actividad en aguadas, por medio de trampas-cámara y observaciones directas.

Material y métodos: Para el análisis con trampas-cámara se utilizaron fotocapturas de pecarí de labios blancos obtenidas en la Evaluación Ecológica Rápida, realizada en el Parque Nacional Mirador-Río Azul en 2004. Asimismo, como parte del presente estudio, en 2006 se estudiaron 12 aguadas, del 22 de abril al 25 de mayo. Durante 2007, se realizaron observaciones directas en las aguadas de febrero a mayo (época seca) de las 8:00 a las 17:00 y se dieron encuentros oportunos en caminos durante junio a enero (época lluviosa). Al observar un grupo, se anotó fecha, hora, número de crías, juveniles y adultos.

Resultados: Durante el 2007, se registró 11 grupos de pecarí de labios blancos. Cinco fueron en aguadas y seis en caminos contabilizando 344 individuos. El tamaño promedio estimado de los grupos, durante la época seca en el Parque Nacional Mirador-Río Azul, fue más grande comparado con la Reserva de la Biosfera de Calakmul y con el Parque Nacional Corcovado. Sin embargo estas diferencias no fueron estadísticamente significativas $(U=9, P=0.08$, $\mathrm{U}=31.5, P=0.63$ ). El $94.2 \%$ de los grupos estuvieron compuestos por adultos; los juveniles representaron el $3.8 \%$ y las crías el $2 \%$. Los grupos visitaron las aguadas con mayor frecuencia entre las 8:00 y 12:00.

Discusión: La media del tamaño de los grupos de pecarí de labios blancos reportados durante la época seca en el Parque Nacional Mirador-Río Azul es más grande que las estimaciones reportadas en estudios en la Reserva de la Biosfera Calakmul, México y en el Parque Nacional Corcovado, Costa Rica. Lo anterior puede deberse al moderado nivel de conservación del Parque Nacional Mirador-Río Azul, a la alta conectividad con áreas protegidas de México y Belice, y a la alta disponibilidad de agua por la presencia del Río Azul. Este río brinda disponibilidad de agua superficial, incluso durante la época seca. Los grupos de pecarí de labios blancos visitaron las aguadas principalmente durante el día. Se registraron dos picos de visitas entre las 8:00 hy 12:00. A causa de lo anterior, es necesario aumentar la protección en el Parque Nacional Mirador-Río Azul, sobre todo en las fronteras con México y Belice. Asimismo deben coordinarse acciones en los tres países para preservar el patrimonio natural de estas áreas protegidas.

Palabras Clave: Aguada; observaciones directas; Selva Maya; trampas-cámara; ungulado.

\section{Literatura citada}

Altrichter, M., E. Carrillo, J. Saenz, y T. Fuller. 2001. White-lipped peccary (Tayassu pecari, Artiodactyla: Tayassuidae) diet and fruit availability in a Costa Rican rain forest. Revista de Biología Tropical 49:1183-1192.

Altrichter, M., y G. Boaglio. 2004. Distribution and relative abundance of peccaries in the Argentine Chaco: associations with human factors. Biological Conservation 116:217-225.

Altrichter, M. 2005. The sustainability of subsistence hunting of peccaries in the Argentine Chaco. Biological Conservation 126:351-362.

Altrichter, M., A. Taber, H. Beck, R. Reyna-Hurtado, L. Lizarraga, A. Keuroghlian, y E. Sanderson. 2012. Range-wide declines of a key Neotropical ecosystem architect, the Near Threatened white-lipped peccary Tayassu pecari. Oryx 46:87-98.

Baur, E. 1999. Estudio de la Cacería de Subsistencia en la Concesión Forestal de Carmelita, San Andrés Peten. Informe Final no publicado, Propeten / Conservation International. Flores, Peten, Guatemala. Informe interno.

Beck, H. 2004. Seed Predation and Dispersal by Peccaries throughout the Neotropics and its Consequences: a Review and Synthesis. Department of Biology, University of Miami. Coral Gables 6:77-115.

Bodmer, R., R. Aquino, P. Puertas, C. Reyes, T. Fang, y N. Gottdenker. 1997. Manejo y Uso Sustentable de Pecaríes en la Amazonía Peruana. Ocasional Paper of the UICN Species Survival Comisión No. 18. UICN-Sur, Quito, Ecuador y Secretaría CITES. Ginebra, Suiza.

Bowkett, A. E., F. Rovero, y A. R. Marshall. 2007. The use of camera trap data to model habitat use by antelope species in the Udzungwa Mountain forest, Tanazania. African Journal of Ecology 46:479-487. 
Congreso de la República de Guatemala. 2004. Ley General de Caza. Decreto número 36-04. Ciudad de Guatemala, Guatemala.

Consejo Nacional de Áreas Protegidas (CONAP). 1995. Ley de áreas protegidas y su reglamento: Decreto 4-89. Guatemala. Ciudad de Guatemala, Guatemala.

Consejo Nacional de Áreas Protegidas (CONAP). 2000. Listado de especies de fauna Silvestre amenazadas de extinción. (Lista Roja de Fauna). Resolución secretaria del CONAP ALC/032-99. Documento de Políticas y Normativos No. 10. CONAP, IDEADS, PROARCA-CAPAS. Ciudad de Guatemala, Guatemala.

Consejo Nacional de Áreas Protegidas (CONAP), y Organización Nacional para la Conservación y el Ambiente (ONCA). 2002. Plan Maestro 2002-2006, Parque Nacional Mirador-Río Azul. Ciudad de Guatemala, Guatemala.

Convention on International Trade in Endangered Species of Wild Fauna and Flora (CITES). 2006. Consultada el 14 de enero de 2014. Disponible en: http://www.cites.org

Cuellar, E., y A. Noss. 2003. Mamíferos del Chaco y de la Chiquitana de Santa Cruz, Bolivia. Editorial Fan. Santa Cruz de la Sierra, Bolivia.

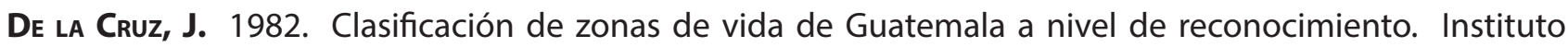
Nacional Forestal, Ministerio de Agricultura, Ganadería y Alimentación. Ciudad de Guatemala, Guatemala.

Estrada, N. 2005. Selección de hábitat y actividad diaria del chancho cariblanco (Tayassu pecari) en el Parque Nacional Corcovado: uso de trampas-cámara. Tesis de Maestría. Universidad de Costa Rica. Heredia, Costa Rica.

Fragoso, J. 1997. Desapariciones locales del baquiro labiado (Tayassu pecari) en la Amazonía: migración, sobre-cosecha o epidemia? Pp. 309-312 in Manejo de fauna silvestre en la Amazonía (Fang, T., R. Bodmer, R. Aquino, y M. Valqui, eds.). United Nations Development Program-Global Environmental Facility, Universidad de Florida, Instituto de Ecología. La Paz, Bolivia.

Fragoso, J. 1998. Home range and movement patterns of white-lipped peccary (Tayassu pecari) herds in the Northern Brazilian Amazon. Biotropica 30:458-469.

García, R., y J. Radachowsky. 2004. Evaluación ecológica rápida del Parque Nacional Mirador Río Azul, Petén, Guatemala. Informe interno, Wildlife Conservation Society, Programa para Guatemala. Flores, Petén, Guatemala.

GruBb, P. 2005. Family Tayassuidae. Pp. 643-644 in Mammal species of the World (Wilson, D., y D. Reeder, eds.). Third Edition. The Johns Hopkins University Press. Baltimore, EE.UU.

Keuroghlian, A., Eaton, D., y W. Longland. 2004. Area use by white-lipped and collared peccaries (Tayassu pecari and Tayassu tajacu) in a tropical forest fragment. Biological Conservation 120:411-425.

Keuroghlian, A., A. Desbiez, R. Reyna-Hurtado, M. Altrichter, H. Beck, A. Taber, y J. M. Fragoso. 2013. Tayassu pecari. IUCN Red List of Threatened Species. Consultada el 10 de marzo de 2014. Disponible en: http// www.iucnredlist.org

Kiltie, R., Y J. Terborgh. 1983. Observations on the behavior of rain forest peccaries in Perú: Why do whitelippped peccaries form herds? Zeitschrift fuer Tirpssychol 62:241-255.

Maffei, L., E. Cuellar, y A. Noss. 2002. Uso de trampas-cámara para la evaluación de mamíferos en el ecotono Chaco-Chiquitanía. Revista Boliviana de Ecología 11:55-65.

MarCH, I. 1993. The white lipped peccary (Tayassu pecari). Pp. 13-22 in Pigs, peccaries and hippos: status survey and conservation plan, IUCN (Oliver, W., ed.). UICN Gland, Suiza.

Mittermeier, R., N. Myers, J. Thomsen, G. Da Fonseca, y S. Olivieri. 1998. Biodiversity Hotspots and Major Tropical Wilderness Areas: Approaches to Setting Conservation Priorities. Conservation Biology 12:516-520.

Morales, J. 1993. Caracterización Etnozoológica de la Actividad de Cacería de la Comunidad de Uaxactún, Flores, El Petén. Tesis de Licenciatura. Universidad de San Carlos de Guatemala. Ciudad de Guatemala, Guatemala. 
Moreira-Ramírez, J., y D. Juárez. 2004. Comparación de Comunidades de Mamíferos Medianos y Mayores en la Parte Alta de la Cuenca del Río Hato (Reserva de Biosfera Sierra de las Minas). Informe interno. Fundación Defensores de la Naturaleza. Ciudad de Guatemala, Guatemala.

Moreira-Ramírez, J. 2009. Patrones diarios de actividad, composición, tamaño y abundancia relativa de manadas de jabalí Tayassu pecari (Link, 1795), en el Parque Nacional Mirador-Río Azul, Petén, Guatemala. Tesis de Licenciatura. Universidad de San Carlos de Guatemala. Ciudad de Guatemala, Guatemala.

Moreira-Ramírez, J., y R. García. 2011. Uso de trampas cámara digitales para estudiar al Jabalí (Tayassu pecari) en el Biotopo Protegido Dos Lagunas, Reserva de la Biosfera Maya. Suiform Soundings 10:32-37.

Myers, N., R. Mittermeier, C. Mittermeier, G. Da Fonseca, y J. Kent. 2000. Biodiversity hotspots for conservation priorities. Nature 403:853-858.

Naranjo, E. J. 2002. Population ecology and conservation of ungulates in the Lacandon Forest, México. Tesis de doctorado. Universidad de Florida. Gainesville, EE.UU.

O’Brein, T., M. F. Gkinnarid, y H. T. WiBisono. 2003. Crouching tigers, hidden prey: Sumatran tiger and prey populations in a tropical forest landscape. Animal Conservation 6:131-139.

Radachowsky, J., V. H. Ramos, R. McNab, y N. Kazakov. 2012. Forest concessions in the Maya Biosphere Reserve, Guatemala: A decade later. Forest Ecology and Management 268:18-28.

Ramos, V., L. Burgués, L. Fleco, B. Castellanos, C. Albacete, G. Paiz, P. Espinosa, y J. Reid. 2007. Análisis económico y ambiental de carreteras propuestas dentro de la Reserva de la Biosfera Maya. Conservation Strategy Fund, Wildlife Conservation Society, Trópico Verde. Serie Técnica No. 8. Flores, Petén, Guatemala.

R Development Core Team. 2011. R: A language and environment for statistical computing. R Foundation for Statistical Computing. Vienna, Austria. http://www.R-project.org.

Reid, F. 1997. A field guide to the mammals of Central America and Southeast Mexico. Oxford University Press. New York, EE.UU.

Reyna-Hurtado, R. 2007. Social Ecology of White-Lipped Peccary (Tayassu pecari) in Calakmul forest, Campeche, México. Tesis de Doctorado. Universidad de Florida. Gainesville, EE.UU.

Reyna-Hurtado, R., y G. Tanner. 2005. Habitat Preferences of Ungulates in Hunted and Nonhunted Areas in the Calakmul Forest, Campeche, Mexico. Biotropica 37:676-685.

Reyna-Hurtado, R., y G. Tanner. 2007. Ungulate relative abundance in hunted and non-hunted sites in Calakmul Forest (southern Mexico). Biodiversity and Conservation 16:743-757.

Reyna-Hurtado, R., Rojas-Flores, E., y G. Tanner. 2009. Home range and habitat preferences of whitelipped peccaries (Tayassu pecari) in Calakmul, Campeche, México. Journal of Mammalogy 90:11991209.

Reyna-Hurtado, R., G. O’Farril, D. Sima, M. Andrade, A. Padilla, y L. Sosa. 2010a. Las aguadas de Calakmul, reservorios de fauna Silvestre y de la riqueza natural de México. Biodiversitas 93:1-6.

Reyna-Hurtado, R., E. Naranjo, C. A. Chapman, y G. Tanner. 2010b. Hunting patterns, population density, group size, and conservation of the white-lipped peccary (Tayassu pecari) in the Calakmul region of Mexico. Oryx 44:88-96.

Reyna-Hurtado, R., H. Beck, M. Altrichter, C. A. Chapman, A. Keuroghlian, A. Desbiez, J. Moreira-Ramírez, G. O'Farril, J. Fragoso, y E. Naranjo. En revisión. What ecological and anthropogenic factors affect group size in white-lipped peccaries (Tayassu pecari)? Biotropica.

RouING, G. 1995. Programa Piloto de Manejo de Vida Silvestre de ARCAS/UICN/CONAP/ USAC-Escuela de Biología. Flores, Petén, Guatemala. Informe interno.

Rosales-Meda, M., M. Hermes, y J. Morales. 2010. Caracterización de la cacería de subsisténcia en comunidades Maya-Q'eqchi'del área de influencia del Parque Nacional Laguna Lachuá, Guatemala. Pp. 25-52 en Uso y manejo de Fauna silvestre en el Norte de Mesoamérica (Guerra, M., S. Calme, S. 
Gallina, y E. Naranjo Piñera, eds.). Serie Hablemos de Ciencia y Tecnología. Secretaria de Educación de Veracruz, Instituto de Ecología A. C., El Colegio de la Frontera Sur. Xalapa, México.

Salom-Pérez, R., E. Carrillo, J. Sáenz, y J. Mora. 2007. Critical condition of the jaguar Panthera onca in Corcovado National Park, Costa Rica. Oryx 41:51-56.

Sanderson, E., M. Jaiteh, M. Levy, K. Redford, A. Wannebo, y G. Woolmer. 2002. The human footprint and the last of the wild. Bioscience 52:891-904.

SowLS, L. 1984. The peccaries. Universidad de Arizona Press. Tucson, EE.UU.

Sowls, L. 1997. Javelinas and other Peccaries: Their, Biology, Management, and Use. The Texas A\&M Univertsity Press. College Station, EE.UU.

TAibel, A. 1977. Mammiferi del Guatemala, con speciale riguardo alla regione del Petén, raccolti dal Maggio al Settembre 1932. Atti Societa Italiana di Scienze Naturali, Museo Cívico di Storia Naturale di Milano 118:379-401.

Traill, L. W., C. J. A. Bradshaw, y B. W. Brook. 2007. Minimum viable population size: A meta-analysis of 30 years of published estimates. Biological Conservation 139:159-166.

Wild life Conservation Society (WCS), y Consejo Nacional de Áreas Protegidas (CONAP). 2009. Paisajes de conservación en la Reserva de la Biosfera Maya: actualización a 2009. USAID, EGAT GCPII. Maya Biosphere Reserve Landscape. Guatemala. Informe interno. Flores, Petén, Guatemala.

Sometido: 7 de marzo de 2015

Revisado: 19 de marzo de 2015

Aceptado: 13 de mayo de 2015

Editor asociado: Consuelo Lorenzo 
